\title{
Signal-Noise Interaction in Optical-Fiber Communication Systems Employing Nonlinear Frequency-Division Multiplexing
}

\author{
Maryna Pankratova $\odot,{ }^{1, *}$ Anastasiia Vasylchenkova $\odot,{ }^{1}$ Stanislav A. Derevyanko $\odot,{ }^{2, \dagger}$ \\ Nikolai B. Chichkov® ${ }^{1}$ and Jaroslaw E. Prilepsky ${ }^{1}$ \\ ${ }^{1}$ Aston Institute of Photonic Technologies, Aston University, Aston Triangle, Birmingham B4 7ET, United Kingdom \\ ${ }^{2}$ School of Electrical and Computer Engineering, Ben Gurion University of the Negev, Beer Sheva 84105, Israel
}

(Received 21 December 2019; accepted 14 February 2020; published 8 May 2020)

\begin{abstract}
We address the properties of nonlinear-Fourier-transform (NFT)-based fiber-optic communications systems and, particularly, study how the presence of noise deteriorates the performance of these systems. Similarly to the case of linear Fourier modes evolving independently under the action of chromatic dispersion alone, NFT-based systems employ so-called "nonlinear modes," forming a nonlinear spectrum, as data carriers, and these nonlinear modes evolve independently in an uncoupled manner under the joint action of nonlinearity and dispersion. However, the influence of amplified-spontaneous-emission (ASE) noise on these nonlinear modes is still relatively poorly studied. In this paper, dealing with a continuous nonlinear spectrum, we scrutinize the properties of the effective noise emerging in the nonlinear Fourier domain. We also show that in the transmission stages, where the signal peak power is relatively high (e.g., at the receiver, in back-to-back transmission, or at short distances), the performance of an NFT system is mostly degraded not by the inline ASE noise, but by the imperfections of the digital sampling and the forward and backward NFT algorithms, i.e., by the NFT processing noise.
\end{abstract}

DOI: 10.1103/PhysRevApplied.13.054021

\section{INTRODUCTION}

Optical-fiber systems are nowadays arguably the most significant part of the global telecommunication network, carrying the bulk of the world's data traffic $[1,2]$ and serving as a fundamental component of information and communication science and technology [3-5]. Taking into account the efficient techniques for compensating fiber losses that exist today, such as lumped or distributed amplification schemes $[1,6]$, the performance quality of an end-to-end optical transmission system in the physical layer is ultimately determined by the interplay of three key phenomena that coexist in a fiber: chromatic dispersion, nonlinear self-action of light due to the Kerr effect, and optical noise, which appears due to the amplification. Even though there exist other, more technical, problems such as optoelectronic signal-conversion-related corruption, insufficient sampling rate, bandwidth limitations, higher-order fiber effects, and the coupling of polarization states, the

\footnotetext{
*pankratovamari@gmail.com

${ }^{\dagger}$ stasd@bgu.ac.il
}

Published by the American Physical Society under the terms of the Creative Commons Attribution 4.0 International license. Further distribution of this work must maintain attribution to the author(s) and the published article's title, journal citation, and DOI. above three factors are largely agreed to be the main fundamental obstacles to the development of high-capacity optical fiber systems [7]. While in the current generation of optical transmission lines the dispersive signal spreading can be compensated by means of digital signal processing (DSP) at the receiver and/or the transmitter, the mitigation of noise and nonlinearity remain the paramount subjects in the development of efficient high-capacity optical transmission systems [3-5]. Yet most of the current optical networks still rely massively on techniques and compensation algorithms that were originally developed and optimized for linear (e.g., radio or wireless) channels. However, the inevitable presence of the nonlinear response of fiber media (first and foremost the Kerr effect) leads to several unwanted phenomena, necessitating their compensation. Thus, in the traditional paradigm, nonlinearity is assumed to have only a detrimental impact on such systems. Over recent years, several solutions for the compensation of nonlinear distortion have been proposed, including digital backpropagation [8], Volterra-type equalization [9], optical [10] and twin-wave [11] phase conjugation, to mention a few important advances; see Ref. [12] for a comprehensive review of the subject. One of the most recent methods of combating nonlinear signal impairments is a technique often referred to as eigenvalue communications or nonlinear frequency-division multiplexing $[13,14]$. This technique involves the transmission of information 
by employing the nonlinear modes provided by nonlinearFourier (NF)-based signal decomposition, such that in this approach the essentially nonlinear characteristics of the signal are used for data modulation [14-16]. This new method, operating with a modulation implemented in the NF domain, has attracted a great deal of attention in recent years; see the review paper [17] and recent studies [18-21]. The approach exploits the fact that the basic master-channel model governing light propagation in a noiseless and lossless single-mode fiber - the nonlinear Schrödinger equation (NLSE) - is integrable, i.e., it can be solved through applying the nonlinear Fourier transform (NFT) provided that some constraints are imposed on the input profile form [22]. The method of solution of the NLSE via the NFT is similar to what we have when utilizing the conventional Fourier transform (FT) to find the solution in the case of linear dynamical systems. We note here that there exist many brilliant monographs explaining the different aspects of the integrability notion in detail and, in particular, its application to the NLSE $[23,24]$.

\section{NFT IN OPTICAL COMMUNICATIONS, AND PROBLEM STATEMENT}

The reason why the NFT can be efficient for the mitigation of effects occurring due to Kerr nonlinearity in an optical channel is that NFT-based transmission methods transform the NLSE into a linear effective channel defined in the NF domain. To explain the concept, we note first that the solution to any integrable evolutionary equation by means of the NFT involves three basic steps [[24], Fig. 4.1]. Let us assume that at the initial point, say at distance $z=0$, we are given a localized input profile. In the first step, we map this input profile into the NF domain (in the linear case, this operation would correspond to the conventional FT and mapping into the frequency domain). This forward NFT operation amounts to the solution of a special direct scattering problem [22,23]; more details are given in Sec. III. As the solution of the aforementioned problem, we obtain a set of scattering data - the parameters of our nonlinear modes. These data, in the most general case, contain the continuous part of the spectrum and a set of localized complex eigenvalues with associated norming constants (also known as spectral amplitudes), altogether collectively known as the NF spectrum or nonlinear spectrum for short. The second stage in the solution procedure is the propagation of the initial NF spectrum obtained in the previous step up to some desired distance, say $z=L$. An important feature of the NFT is the fact that the NF modes evolve in a linear decoupled way, with each nonlinear spectral component acquiring a phase shift defined by the dispersion law of the progenitor linearized system, so that the evolution of the NF spectrum is trivial. We note that, at the same time, the physical space-time evolution of a pulse can be quite complex. In the final stage, we recover the profile of our solution in the time domain. To do so, in the linear case one simply applies the inverse FT. In the nonlinear case, an effective inverse NFT is performed by means of solving the so-called Gelfand-Levitan-Marchenko equations [22,23], and, as the result, we find a solution to our integrable equation at distance $L$.

These three stages that provide the solution of a nonlinear equation at a desired distance constitute the method known by the name "inverse scattering transform" in the mathematical literature [22-24]. Looking at this scheme, one can realize that by applying all the steps above in reverse order we can effectively compensate all the effects of nonlinearity and dispersion at the receiver, thus implementing a NFT-flavored backpropagation [17,25]. However, this technique has not been completely consummated into a practically working concept, mainly because of the difficulties in dealing with a possibly very complex NF spectrum structure emerging from conventional randomly coded signals [26], together with the requirement for localization of the signal (in contrast to the continuous streams used in "linear" methods) and the limited capability of the most current NFT methods for processing very long (highenergy) signals $[27,28]$. A more sophisticated use of the NF domain was proposed in Ref. [14] (the idea goes back to the now well-recognized work of Hasegawa and Nyu [13]), where one multiplexes the signals not in the conventional Fourier frequency domain but in the NF domain. This technique was termed nonlinear frequency-division multiplexing (NFDM), by analogy with conventional linear wavelength-division multiplexing (WDM). Nonlinear multiplexing means that instead of allocating linear frequency bands for individual users as we do within the traditional WDM concept, for NFDM we now allocate regions of the nonlinear spectrum for the same purpose. An important aspect of the NFT [13] is that due to the effective linearization of the channel, all nonlinear propagation effects such as self-phase modulation, cross-phase modulation, and four-wave mixing are effectively absent owing to the orthogonality property of the components of the NF spectrum (provided that all the channels are nonlinearly multiplexed). In engineering terms, the NFT method can be treated as a nonlinear analogue of orthogonal frequency-division multiplexing [14].

While having the potential to overcome the nonlinearityimposed signal degradation, NFDM still relies on the integrability property of the underlying propagation model. This means that when using NFT-based signal processing, we inherently assume that the space-time channel is exactly the unperturbed NLSE. While the gain and loss budget (in both lumped and distributed cases) can be approximately accommodated within the same NLSE framework using, e.g., the so-called path-average approximation [29-33], amplified spontaneous emission (ASE) always remains a detrimental factor, and leads to signal 
distortion and signal-noise beating both in the time domain and in the linear or nonlinear spectral domain. Fortunately, in most important and physically relevant situations the noise can be treated perturbatively, which makes it possible to describe NFDM transmission as a first-order approximation of an integrable model corrupted by a stochastic inline perturbation. However, as of today, results concerning the effect of ASE on the nonlinear spectrum are still few and far between, encompassing mostly special cases and specific simplifying approximations, while a complete thorough study of signal-noise beating for signals employing the full nonlinear spectrum is largely missing. In this context we mention here, first, studies addressing the celebrated Gordon-Haus effect $[33,34]$, i.e., the random jitter of soliton parameters corresponding to the case of a NF spectrum containing a single discrete eigenvalue (a single soliton); some further results can be found in Refs. [35-37]. More recent results concerning the effect of noise on NFDM systems employing more than one discrete NF mode can be found in Refs. [38-40] and references therein. Also, one should mention Ref. [41], where the statistics of the scattering NF coefficients emerging in the discretized NFT were computed for a signal contaminated by additive white circular Gaussian noise, though the dynamics was not studied there. Within the same framework, one can compute the statistics of the continuous nonlinear spectrum in the case when the deterministic component of the signal is negligible compared with symmetric Gaussian noise [42].

In recent publications [40,43], some of the present authors derived an analytical model for the statistical properties of the nonlinear spectral noise emerging in NFDM systems utilizing the continuous part of the NF spectrum, where first-order perturbation theory with respect to the signal-noise ratio (SNR) was used. (Reference [40] also contains the perturbation expressions for the general case when all types of NF components are present.) It was predicted that the spectral density of this continuous nonlinear spectral noise scales as a low-order polynomial of the initial spectral power: in other words, the noise emerging in the NF domain is strongly signal-dependent. Similar results were also obtained later in Ref. [44], where a somewhat different approach was used. However, these results have not been thoroughly tested directly, and neither have the spectral properties of the nonlinear noise been studied numerically for long-haul transmission lines employing NFDM. (An indirect comparison of the model results in Ref. [43] with numerical tests in terms of $Q$-factor dependences can be found in Ref. [[45], Fig. 2(a)].)

The current paper is aimed at filling this gap. We report results concerning the statistical properties of nonlinear spectral noise and show that the actual levels of such noise appear to be higher than those predicted by the theory of Ref. [43]. We identify and emphasize the source of this excess noise as the error caused by insufficient sampling in both the time and the frequency domain and by the clipping of the signal by the finite processing window, which is especially prominent for short-reach systems. We call this type of impairment processing noise as its source is the finite accuracy of the numerical NFT operations used for signal processing, and, as such, it is independent of the external ASE. Here, we mention that there exist several studies where the performance degradation of NFDM systems is attributed almost solely to growing signal-noise interactions [45-47]. Indeed, the increased signal-noise beating for high values of the input signal power was actually predicted by our earlier theoretical studies [43], where no noise sources other than ASE were taken into account. However, some authors [27] also mention the discrepancies of such a treatment with several preceding results, and emphasize the importance of the errors arising due to features of NFT-based processing, arguing that the latter can be the dominating limiting factor in some situations. In our study, we show that this indeed can be the case.

Finally, we note that in this paper we choose to concentrate on the most relevant channel-distortion factors affecting NFDM systems - namely, ASE and numerical noise. However, there exist multiple other sources not taken into account by the idealized model, including but not limited to third-order dispersion, nonuniformity of the gain-loss profile, and the Raman effect [1]. We also do not consider polarization multiplexing. All these effects and generalizations are left for future work, as we concentrate on a simplified system for which there exist simple analytically tractable expressions.

Some preliminary results of this study have been reported in Refs. [48-50].

\section{DESCRIPTION OF A NFT-BASED TRANSMISSION SYSTEM TAKING ACCOUNT OF DISTRIBUTED NOISE}

The details of the NFT operations and their usage can be found in many classic texts (see Refs. $[23,24]$ ), while the use of the nonlinear spectrum for data transmission has been covered in several recent reviews [14,17,21]. However, in order to keep the presentation self-consistent, we provide the basic facts of the subject here.

\section{A. Basics of NFT-based optical transmission approach}

The main channel propagation model considered here is the so-called generalized NLSE, and in this paper we deal with the case of a single polarization (the scalar case). It is also possible to consider polarization-division-multiplexed NFT-based systems where the space-time optical transmission channel is approximated by the so-called Manakov system $[21,51,52]$, which, however, is beyond the scope of this paper. 
For the purposes of the NFT, the generalized NLSE for the envelope electric field function $q(z, t)$ can be written in a standard dimensionless form,

$$
i \frac{\partial q(z, t)}{\partial z}+\frac{\partial^{2} q(z, t)}{\partial t^{2}}+2|q|^{2} q=\eta(z, t),
$$

where $z$ and $t$ are the normalized distance along the fiber and the time in the retarded reference frame, respectively. The normalization units for time, distance, and power are standard and can be found in many references for both idealized distributed and idealized lumped amplification schemes $[1,14,17,43]$. A brief summary of these normalizations is presented in Appendix A. By dropping the rhs in Eq. (1), one recovers a standard integrable NLSE amenable to an NFT-based exact solution [22]. The form of the NLSE (1) with the rhs adopted here takes amplified spontaneous emission into account via the $\eta(z, t)$ term, modeled as a complex white additive Gaussian process with zero mean. The latter is fully characterized by the (normalized) power spectral density (PSD) $2 D$ :

$$
\mathrm{E}\left[\eta(z, t) \eta^{*}\left(z^{\prime}, t^{\prime}\right)\right]=2 D \delta\left(t-t^{\prime}\right) \delta\left(z-z^{\prime}\right),
$$

where, here and below, E[·] denotes the expectation-value operator. Equation (1) assumes ideal distributed Raman amplification with full compensation of the fiber loss, although, as mentioned before, a similar type of model is recovered in the leading approximation in the lumpedamplification case after path averaging [29-31,33]. In both cases, expressions for the PSD intensity $2 D$ of the ASE are readily available (see Ref. [7] and Appendix A), and the difference amounts merely to the effective value of the nonlinearity coefficient [29].

The forward NFT operation consists in solving the socalled Zakharov-Shabat scattering problem for a pair of auxiliary functions $\phi_{1,2}(t)$ [22]:

$$
\frac{d \phi_{1}}{d t}=q(t) \phi_{2}-i \xi \phi_{1}, \quad \frac{d \phi_{2}}{d t}=-q^{*}(t) \phi_{1}+i \xi \phi_{2} .
$$

Here $\xi$ is a (generally complex) eigenvalue (the nonlinear analogue of the Fourier frequency), and the pulse $q(t)$ (for brevity, we have omitted the explicit dependence on the distance $z$ ) is our field to be decomposed; the asterisk means complex conjugation. In the NFDM concept, the information-bearing signal is surrounded by sufficiently large zero-padding guard bands, usually taken equal to the linear dispersive spread of the signal (i.e., to the dispersion-induced memory), and thus one can assume our signals to be of finite support. In other words, NFDM systems of the type considered here always operate in a burst mode $[17,29,53]$. In this paper we employ only the continuous NF spectrum, similarly to the systems considered in Refs. [15,29,43,53], and no discrete spectrum (soliton modes) is present, so that the parameter $\xi$ is assumed real. A hybrid transmission method that employs both parts of the spectrum has also been reported in the literature [16], but we leave the full study of the noise properties of such systems for later (although some preliminary results were reported in [50]). For the forward NFT operation, we define a specific solution of Eq. (3), $\Phi(t, \xi)=\left[\phi_{1}, \phi_{2}\right]^{T}$, with the "initial" condition at the trailing end of the pulse (assumed here to be at $t=0)\left.\Phi\right|_{t=0}=[1,0]^{T}$. Then, the solution at the leading end of the burst at $t=T$ must necessarily take the form $\Phi_{t=T}=\left[a(\xi) e^{-i \xi T}, b(\xi) e^{i \xi T}\right]^{T}$, where the functions $a(\xi)$ and $b(\xi)$ are called scattering coefficients and constitute the core of NFT-based pulse decomposition. The continuous part of the nonlinear spectrum is defined by the ratio of these scattering coefficients,

$$
r(\xi)=b(\xi) / a(\xi),
$$

widely known as the reflection coefficient. In the limit of small powers, $|q| \ll 1$, the reflection coefficient is simply related to the linear Fourier spectrum $\mathcal{F}(\omega)$ via [23]

$$
\begin{aligned}
r(\xi) & =-\int_{-\infty}^{\infty} q^{*}(t) e^{-2 i \xi t} d t+O\left[|q|^{2}\right] \\
& =-\left.\mathcal{F}^{*}(\omega)\right|_{\omega=2 \xi}+O\left[|q|^{2}\right] .
\end{aligned}
$$

Several NFT schemes for encoding the data directly on the continuous spectrum have been considered in the literature. One of them is the so-called nonlinear inverse synthesis (NIS) [15], where the nonlinear spectrum $r(\xi)$ coincides up to a trivial scaling factor with a linear FT of some conventional modulated signal. Another flavour of the same method is called $b$-modulation and uses the same principle but for the coefficient $b(\xi)$ rather than $r(\xi)$ [54,55], but we do not address this type of NFDM here. We also do not use NFT precompensation at the transmitter as was done, e.g., in Ref. [56], because our goal here is not to get the highest spectral efficiency possible but rather to understand how noise corrupts our data.

In the NIS scheme, we start from the continuous NF spectrum and then generate the respective time-domain profile, insofar as the inverse NFT provides a unique mapping from the NF spectrum to the corresponding localized time-domain signal $[22,23]$. We also assume that no discrete spectrum components appear during the propagation or that their influence is insignificant (initially they are absent by design).

The inverse NFT mapping performed at the transmitter gives us the initial waveform $q(t, 0)$ (in dimensionless units). Then the signal obtained is rescaled to real-world units and the generated pulse is launched into the optical fiber. At the receiver, located at a normalized distance 


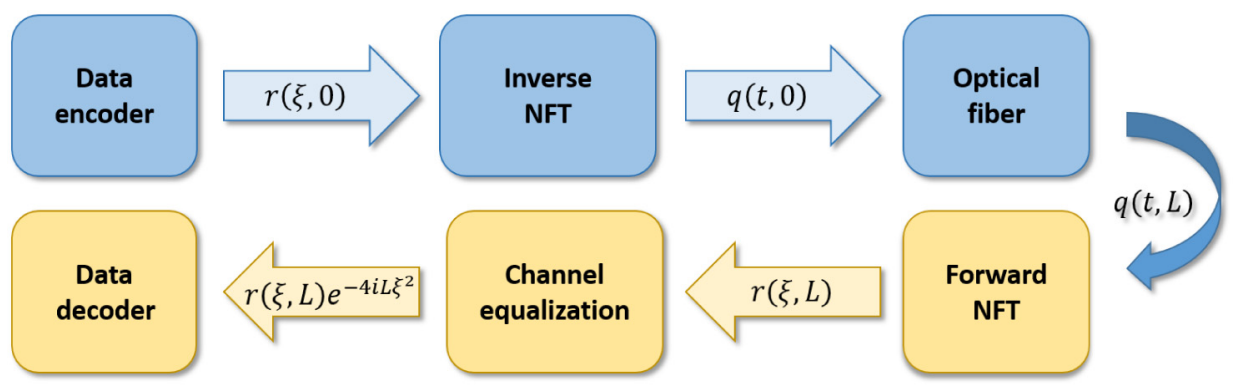

FIG. 1. Basic elements of a NFDM optical communication system, which, in our case, is based on nonlinear inverse synthesis with utilization of inverse NFT (INFT) and NFT operations for signal processing at the transmitter and receiver.

$z=L$, we perform the forward NFT operation, retrieving the data-bearing continuous spectrum component and removing the accumulated phase rotation by inverting the following relation (a single-tap channel equalization):

$$
r(\xi, z=L)=e^{4 i \xi^{2} L} r(\xi, z=0) .
$$

A schematic illustration of the transmission stages is given in Fig. 1.

\section{B. Effective noise in the NF domain}

Regardless of the particular NFDM scheme used, the presence of distributed noise in the rhs of Eq. (1) breaks the exact integrability and adds a stochastic component to the evolution of the NF spectrum $r(\xi, z)$ [40,43]. In the regime where the perturbation is small and the system is close to an integrable one, this effective noise in the NF domain (after postcompensation) can be modeled as an effective additive term $\Gamma(\xi, z)$, defined via the relation

$$
e^{-4 i \xi^{2} L} r(\xi, L)=r(\xi, 0)+\Gamma[\xi, r(\xi, 0)] .
$$

Here we note an important point: this effective complex noise term $\Gamma$ in Eq. (5) has two main independent contributions coming from different sources. The first one, which we denote $\Gamma$, is physical (noise proper) and comes from the above-mentioned influence of the inline ASE noise in the time domain on the NF spectrum. The second source of spectral distortion comes from the finite accuracy of the direct and inverse NFT methods, which, in turn, rely on finite sampling of the signal. This noise is present even in a purely integrable system and represents a limitation on the accuracy of our sampling and integration methods both for the direct NFT given by Eq. (3) and the INFT based on solving the Gelfand-Levitan-Marchenko equations. In this paper, we call this noise component processing noise and denote it by $\Gamma_{\text {proc }}$.

We begin here by summarizing a few theoretical results known about the first (physical) noise component $\Gamma$ emerging due to ASE. These results are mostly taken from our previous work [43]. The first important property of this noise is that it is input-dependent, which makes the communication channel [Eq. (5)] noticeably distinct from the trivial additive-Gaussian-noise channel. Secondly, the two quadratures of the complex noise are not symmetric [in contrast to its optical-domain progenitor, $\eta(z, t)$ in Eq. (1)], which means that apart from the covariance function $\mathrm{E}\left[\Gamma(\xi) \Gamma^{*}\left(\xi^{\prime}\right)\right]$, for the complete characterization of that process we must also consider the pseudocovariance $\mathrm{E}\left[\Gamma(\xi) \Gamma\left(\xi^{\prime}\right)\right]$. In Ref. [43], approximate leading-order expressions were derived for both the covariance and the pseudocovariance of the NF-domain noise, assuming ideal distributed amplification, high SNR, and a sufficiently long link length $L$. These expressions have the simple form

$$
\begin{aligned}
\mathrm{E}\left[\Gamma(\xi) \Gamma^{*}\left(\xi^{\prime}\right)\right] & =2 D L \pi \delta\left(\xi-\xi^{\prime}\right) E_{1}(\xi), \\
\mathrm{E}\left[\Gamma(\xi) \Gamma\left(\xi^{\prime}\right)\right] & =2 D L \pi \delta\left(\xi-\xi^{\prime}\right) E_{2}(\xi),
\end{aligned}
$$

where the normalized nonlinear PSD and the pseudodensity $E_{1,2}(\xi)$ explicitly depend on the effective "power" in the NF domain. These explicit dependences of densities, obtained in Ref. [43], are as follows:

$$
\begin{aligned}
& E_{1}(\xi)=1+|r(\xi)|^{2}+|r(\xi)|^{4}, \\
& E_{2}(\xi)=r(\xi)^{2} .
\end{aligned}
$$

In most of the numerical simulations that we perform later on, the above quantities are additionally averaged over the transmitted symbols $c_{k}$; see Eq. (8) below. Notice that according to the approximate theory of Ref. [43], the nonlinear spectrum is delta-correlated and its effects accumulate linearly with distance similarly to a linear system when the normalized spectrum is small, $|r(\xi)| \ll 1$. In fact, the linear regime corresponds to the approximation $E_{1} \approx 1$ and $E_{2} \approx 0$. The complex interaction of signal and noise in the NF domain is marked by the dependence of both densities on the input. In Ref. [44], somewhat similar expressions were obtained for $E_{1,2}(\xi, z)$, and the highest order in the polynomial dependence of the density on the input was $z$-dependent.

However, the theoretical approach used for deriving the results in Eqs. (6a)-(7b) makes a few assumptions about the nature of the input and the parameters of the system that are not obviously met, especially at high signal powers. Indeed, our preliminary results [48-50] indicate that in some situations we can have noticeable deviations from the theoretical results given in Ref. [43]. In this paper, 
we attribute these deviations to the processing noise and complete this study to reveal the characteristics of the communication system and the signal itself which affect this deviation.

\section{SIMULATIONS AND RESULTS}

\section{A. Description of transmission system}

In our study here, we use the popular sinc spectral shape of subcarriers, $\operatorname{sinc}(y)=\sin \pi y /(\pi y)$, which corresponds to the NFT analogue of orthogonal frequency-division multiplexing (OFDM) $[53,57]$. Then, the NF spectrum (the reflection coefficient) is modulated in the following way:

$$
r(\xi, 0)=\sum_{k=-N_{\mathrm{ch}} / 2+1}^{N_{\mathrm{ch}} / 2} c_{k} \operatorname{sinc}(2 \xi-k),
$$

where $c_{k}$ are randomly taken from the chosen modulation alphabet defining the particular constellation shape, and $N_{\text {ch }}$ is the number of subcarriers (channels). This OFDMtype modulation of the NF spectrum has been widely used $[15,16,29,53]$, and was also addressed theoretically in Ref. [43]. Such a spectral shape is convenient for NFDM transmission because $r(\xi)$ in Eq. (8) is a band-limited function, such that its linear FT has a finite extent; the latter is important when forming well-localized bursts in the true time domain to preclude the overlapping of symbols and detrimental effects resulting from intersymbol interference. We simulate the transmission of the optical signal in a single-mode fiber with standard parameters [53,57] for a range of link lengths (up to $2000 \mathrm{~km}$ ). All the results in our paper are obtained by averaging over 600 realizations of randomly selected sequences of 64 or 128 quadraturephase-shift-keying (QPSK) subcarriers, used in the input profile of Eq. (8); the latter numbers are typical values for similar systems $[29,53]$. We assume ideal distributed Raman amplification in the simulations, also adopting the corresponding properties of the ASE noise, and use explicitly the model of Eq. (1). The spectral noise in the NFT domain is extracted at the receiver using Eq. (5). In all our simulations, the dimensionless PSD of the ASE term is chosen to be $2 D=0.04$. According to Appendix A, this corresponds to standard telecommunication values and to a total bandwidth of $100 \mathrm{GHz}$, a photon occupancy factor $K_{T}=1.3$, and a fiber loss of $0.2 \mathrm{~dB} / \mathrm{km}$.

To study the properties of the effective noise, we use the parameter $S=\left|c_{k}\right|^{2}$ as a measure of the effective signal power (per subcarrier) in the NF domain. For the QPSK format, all symbols are allocated the same power and no ensemble averaging is required. Note that this parameter is related in a rather nonlinear way to the signal power and energy in the optical domain (see the discussion and figures in Supplemental Material for Ref. [43]). Here we study a wide range of NF signal powers, from low values representing the almost linear limit to powers in the vicinity of the system-performance maximum, $S \approx 0.87$ [see the explanation for this value in the next subsection, after Eq. (10)].

\section{B. Results of Monte Carlo simulations}

We begin by presenting an overall two-dimensional density plot of the covariance and pseudocovariance matrices [Eqs. (6a) and (6b)], shown in Fig. 2 for fixed values of the signal power per subcarrier, $S$, and the total propagation distance, $L$. The results are obtained by averaging over 600 realizations of the initial symbols and noise. One can readily observe that the diagonal elements are dominant, in agreement with the leading-order analytical expressions in Eq. (6a). Also, the averaged pseudocovariance seems to be washed out (note the scales on the color bars), which is again in line with the theoretical prediction in Eq. (6b), which (unlike the covariance) is phase-sensitive and should vanish when averaged over any symmetric constellation.

The results in Fig. 2 represent the typical appearance of the covariance matrix that we observe for all values of power, distance, and subcarrier number studied. The main difference between these results is in the value of the diagonal elements, which is proportional to the nonlinear PSD, which, according to theory [Eq. (6a)] and our simulations, grows with the signal power and propagation distance.

Let us study further the properties of the diagonal elements of the pseudocovariance and covariance matrices (i.e., densities and pseudodensities) as functions of the signal power and propagation distance. In Figs. 3 and 4 , we present the results for the total nonlinear power spectral density, $\mathcal{E}_{1}$, and the absolute value of the pseudodensity, $\left|\mathcal{E}_{2}\right|$, extracted from numerical simulations via Eqs. (5)-(6b) by averaging over the inline noise, the input symbol realizations, and the nonlinear bandwidth. The latter averaging, in the NF domain, is performed as follows:

$$
\mathcal{E}_{i}=\frac{1}{W_{\mathrm{NF}}} \int_{-W_{\mathrm{NF}} / 2}^{W_{\mathrm{NF}} / 2} E_{i}(\xi) d \xi, \quad i=1,2,
$$

where $W_{\mathrm{NF}}$ is the nonlinear bandwidth determined from Eq. (8). We stress here that we average the normalized quantities, i.e., we divide the result by the factor $2 D L \pi$, which corresponds to the linear PSD, and, according to the theoretical predictions of Eq. (6), these quantities should not depend on the distance $L$. The spectral averaging is performed in order to obtain a uniform smooth measure of the accumulated ASE noise transferred into the NF domain. The data are presented for two distinct numbers of subcarriers.

Let us first check how the normalized density and pseudodensity behave for different propagation distances. It can be seen from Fig. 3 that as the system deviates from the 

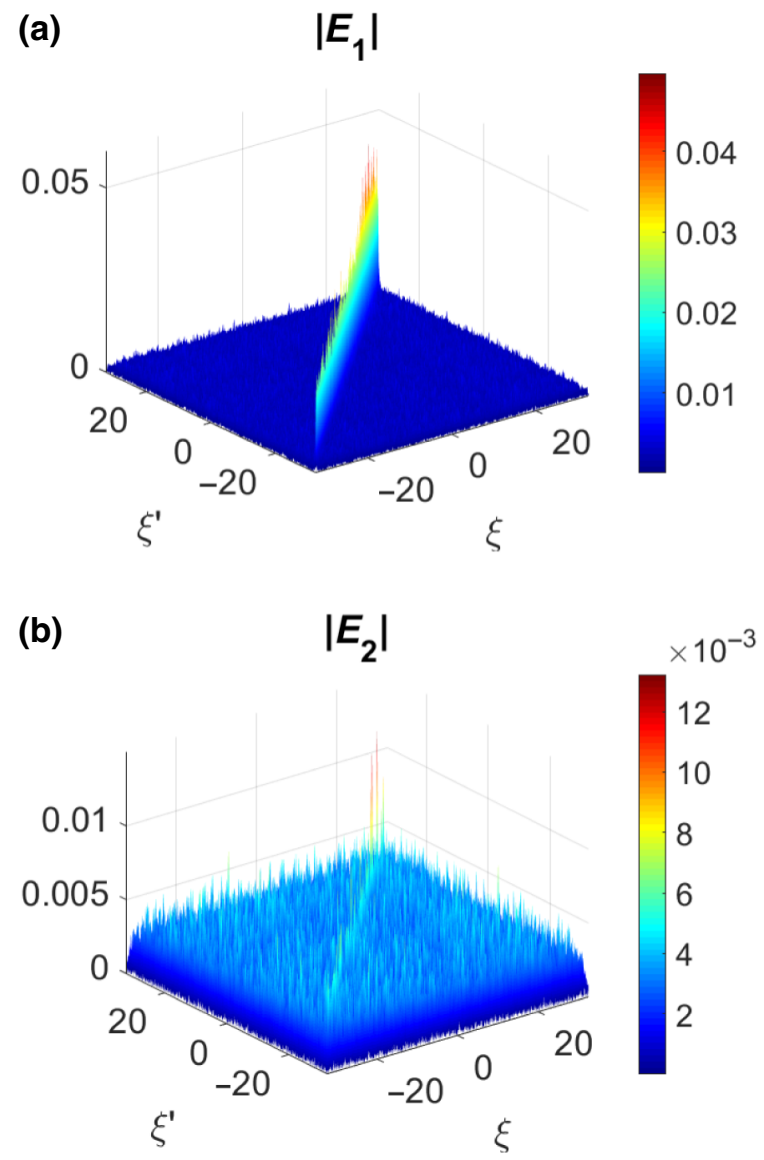

FIG. 2. Absolute values of the covariance $\left|\mathrm{E}\left[\Gamma(\xi) \Gamma^{*}\left(\xi^{\prime}\right)\right]\right|$ (a) and pseudocovariance $\left|\mathrm{E}\left[\Gamma(\xi) \Gamma\left(\xi^{\prime}\right)\right]\right|(\mathrm{b})$, averaged over the initial symbol distribution, as a function of two "nonlinear frequencies" $\xi$ and $\xi$, for fixed nonlinear power per subcarrier $S=0.87$ and distance $L=1000 \mathrm{~km}$, and $N_{\mathrm{ch}}=128$ nonlinear subcarriers.

linear regime (the product $S N_{\text {ch }}$ grows), the averaged density $\mathcal{E}_{1}$ grows significantly compared with its pseudolinear limit $\mathcal{E}_{1}=1$. However, a more interesting observation is that it becomes distance-dependent for larger power values $S$ (and this is especially pronounced for the higher number

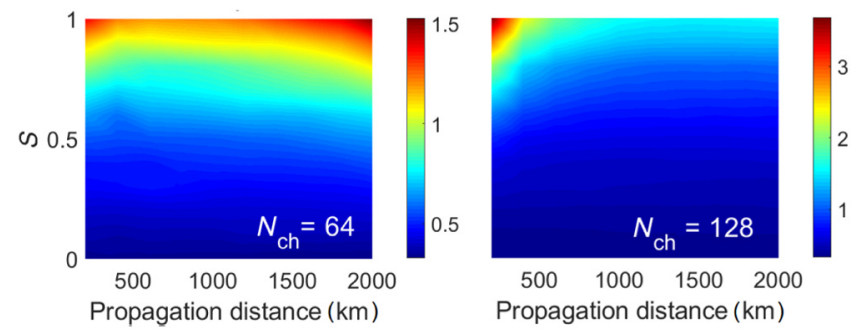

FIG. 3. Dependence of the total noise PSD $\mathcal{E}_{1}$ on the effective power in the NF domain $S$ and propagation distance $L$ for 64 (left) and 128 (right) OFDM subcarriers used in the modulation of the reflection coefficient [Eq. (8)]. of subcarriers in the right pane). For almost every level of the input power, the effective power density $\mathcal{E}_{1}$ is higher at smaller distances, getting closer to its linear asymptotic value in the interim, and slowly growing again at larger $L$.

This behavior is partially explained by the fact that at small distances the asymptotes in Eq. (6), derived in Ref. [43], are no longer valid. But, as we shall see below, the main source of the signal distortion in the high-power and low-distance region is the presence of a numerical error that stems from the fact that the accuracy of both the direct and the inverse NFT procedures decays with an increase in the burst energy [58]. This type of signal distortion is considered below in Sec. IV C and was not taken into account in the theory of Ref. [43]. The contribution of this excess noise, normalized per unit distance and bandwidth [as in Eq. (9)], is maximal for high power and short distance, and the area where one would expect the best NFDM-system performance is defined by the trade-off between the processing noise at short distances and the accumulated ASE noise at large distances.

For a fixed propagation distance $L$, in all the regimes considered here, increasing the power per subcarrier $S$ always leads to an increase in the PSD $\mathcal{E}_{1}$. However, at short distances the growth of the noise power is sharpest, due to the considerable contribution of processing noise, while at higher distances, when the pulses are dispersed within the burst so that the average power drops, it is the ASE component, $\Gamma$, that dominates the PSD.

When it comes to the pseudodensity $\left|\mathcal{E}_{2}\right|$ shown in Fig. 4, we also observe its nontrivial dependence on the propagation distance. However, the averaging over the input washes away much of the variation of the pseudocovariance, and its typical absolute values appear to be at least two orders of magnitude smaller than those for the PSD.

Let us study specific sections of the above diagrams in the $(S, L)$ phase space. To compare the simulation results with the theory, we need to obtain values of the normalized density and pseudodensity [Eq. (7)] averaged over the
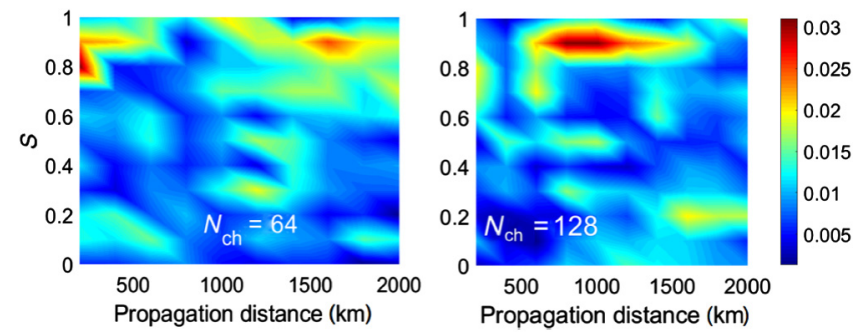

FIG. 4. Dependence of the total noise pseudodensity $\left|\mathcal{E}_{2}\right|$ on the effective power in the NF domain $S$ and propagation distance $L$ for 64 (left) and 128 (right) OFDM subcarriers used in the modulation of the reflection coefficient [Eq. (8)]. 
spectrum [Eq. (9)] and the input symbols $c_{k}$. For the pseudodensity, as mentioned above, one has $\mathcal{E}_{2} \approx 0$ for any symmetric constellation, and for the normalized density we present general results in Appendix B, which, in the case of QPSK considered here, specialize to

$$
\mathcal{E}_{1}(S)=1+S+\frac{4}{3} S^{2}
$$

Note that this result is independent of the propagation distance $L$ (as it enters only the linear value used for normalization) and the number of subcarriers $N_{\mathrm{ch}}$. In the following, we compare the numerical results with this theoretical benchmark. The effective SNR in the NFT domain is given by the ratio $S /\left(2 \pi D L \mathcal{E}_{1} N_{\mathrm{ch}}\right) \propto S / \mathcal{E}_{1}(S)$. This quantity achieves its maximum at an optimal dimensionless input power per subcarrier $S_{\text {opt }}=\sqrt{3} / 2 \approx 0.87$, which we shall use extensively when comparing theory and numerical results. For the case of $N_{\mathrm{ch}}=128$ and a propagation distance $1500 \mathrm{~km}$, given the rest of the transmission parameters this corresponds to a maximum achievable SNR of about $21 \mathrm{~dB}$. We also choose a second characteristic low power level $S=0.005(\mathrm{SNR}=12 \mathrm{~dB})$ as a benchmark of the system performance in the quasilinear regime. Note that a high effective SNR is a necessary but not sufficient condition for the applicability of the perturbative approach, as discussed in Ref. [43].

We start with the dependences on the distance in more detail: we present them in Fig. 5 for specific values of the input effective power per subcarrier $S$. One can see that for higher input power, the theoretical result in Eq. (10) derived for the ASE underestimates the value of the observed NF noise. It can also be observed from Fig. 5 that, depending on the number of subcarriers, $\mathcal{E}_{1}$ can be as much as 5 times higher than its linear limit at short distances, while at a distance of around $600 \mathrm{~km}$ it drops before starting to grow very slowly again. At higher powers, the higher values of $\mathcal{E}_{1}$ occurring at low distances are connected to the processing noise, while at distances above $600 \mathrm{~km}$ the accuracy of the NFT algorithm improves sufficiently due to the overall decay of the signal amplitude, and we see a sharp decrease in $\mathcal{E}_{1}$ [which is more pronounced in Fig. 5(c)], followed by a slight increase with propagation distance.

The absolute averaged value of the pseudodensity $\left|\mathcal{E}_{2}\right|$ seems to correspond to the residual averaging error.

Next, we present the dependence of the spectral density and pseudodensity on the effective power for two fixed values of the propagation distance, addressing the same characteristic values of the number of subcarriers, $N_{\text {ch }}=64,128$. We choose two representative values of the propagation distance to check both short and long distances. Again, the density $\mathcal{E}_{1}$ is compared with the polynomial analytical formula given in Eq. (10) and, as for the pseudodensity $\mathcal{E}_{2}$, the theory again predicts that it should vanish after averaging over the initial symbol distribution.

It can be seen from Figs. 6(a) and 6(c) that the spectral density $\mathcal{E}_{1}$ grows with the power faster than the analytical expression in Eq. (7a) predicts. It is also worth noticing that the difference between the approximate theory and the

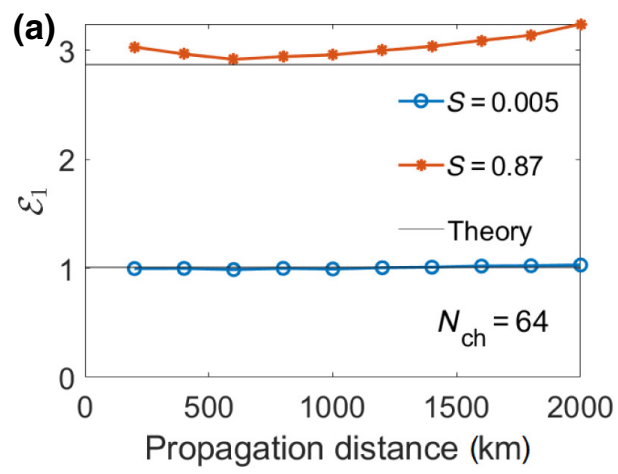

(c)

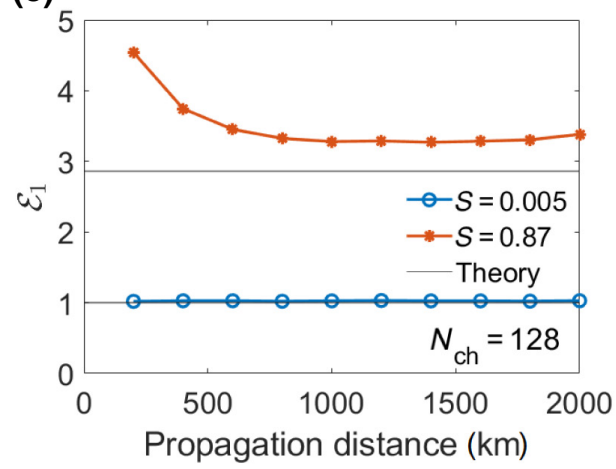

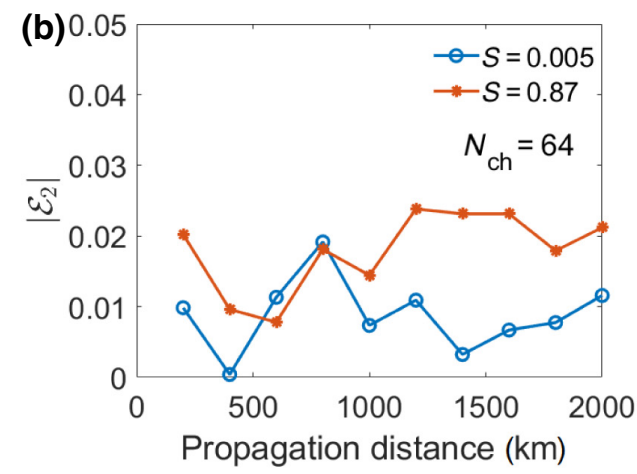

(d)

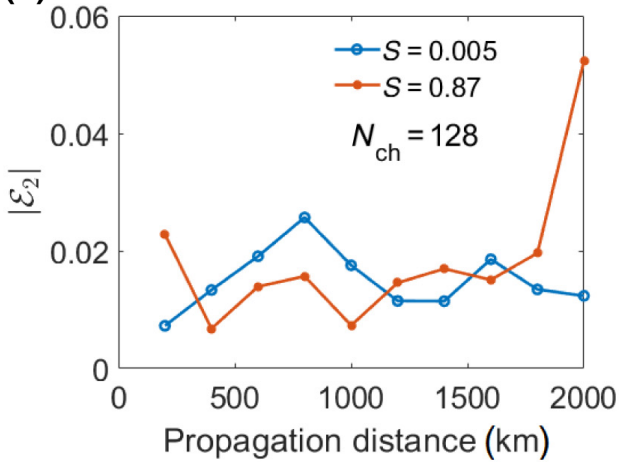

FIG. 5. Dependence of the total noise PSD $\mathcal{E}_{1}$ (a),(c) and pseudodensity $\left|\mathcal{E}_{2}\right|$ (b),(d) on the propagation distance for $64(\mathrm{a}),(\mathrm{b})$ and 128 (c),(d) subcarriers, drawn for two different (low and high) values of the effective NF power $S$. 
(a)

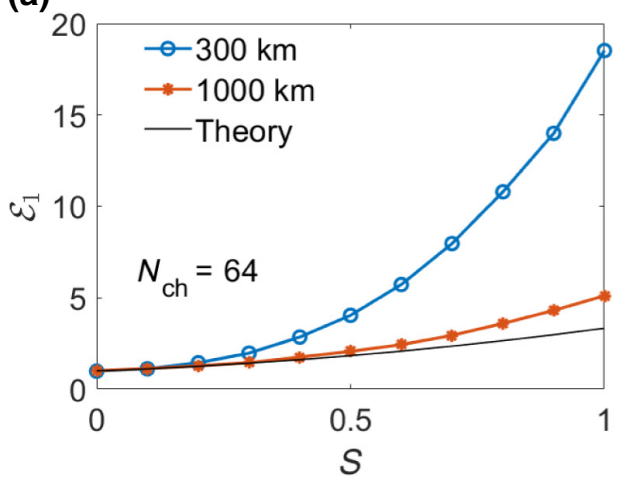

(c)

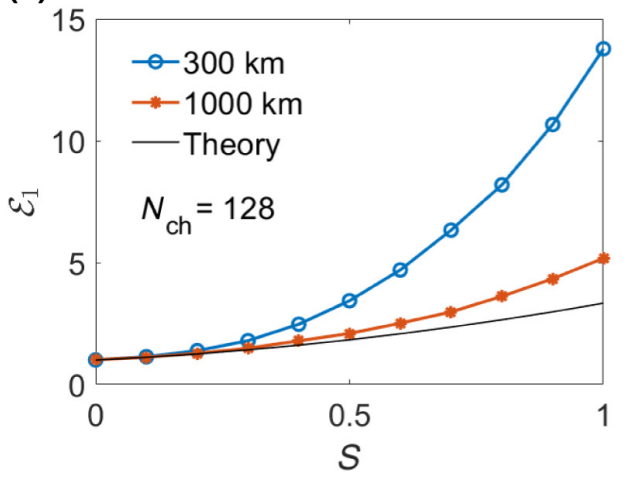

(b)

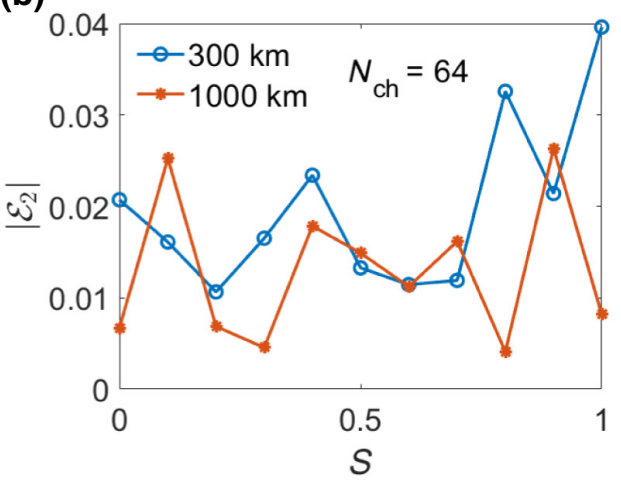

(d)

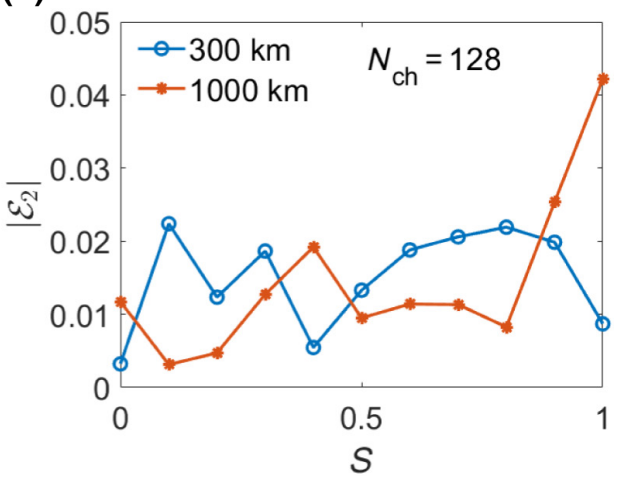

FIG. 6. Dependence of the total power spectral density $\mathcal{E}_{1}$ (a),(c) and pseudodensity $\left|\mathcal{E}_{2}\right|(\mathrm{b})$,(d) on the effective power $S$ for 64 (a),(b) and 128 (c),(d) subcarriers for two propagation distances, 300 and $1000 \mathrm{~km}$. The solid black line denotes the theoretical formula in Eq. (10). numerical data is higher for the larger number of subcarriers and, hence, for a higher overall burst energy in both the NF and the time domain. The latter result is especially pronounced at smaller distances.

One explanation is that in the large-distance region where the ASE contribution dominates, the theory of Ref. [43] assumes a specific large-distance asymptotic form of the Jost functions [the solutions of Eq. (3)] first derived by Zakharov and Manakov [59]. This asymptotic form was explicitly used in Ref. [43] to derive the analytical expressions in Eq. (7). However, the rate of convergence of these asymptotes with distance can potentially be slow for NIS-modulated spectra such as that in Eq. (8), which could produce some disparity with the theory. The nonlinear mixing of part of the processing noise with the signal could also contribute to the observed deviation. Therefore, even after $1000 \mathrm{~km}$, the deviations from the asymptotes and other contributing factors can be noticeable enough for these effects to translate into deviations from the spectral densities observed in our simulations, shown in Fig. 6. But, as we shall see below, the main culprit for the deviation from the theory is the processing noise, which we shall study in the following two sections.

\section{Processing noise}

Now let us turn to studying the processing noise and its contribution to the overall picture. In our simulations, we use fast NFT and INFT algorithms developed by Wahls and collaborators [58,60-63]. These algorithms demonstrate the highest performance-vs-accuracy characteristics: these methods deliver a signal-processing complexity of order $O\left(n \log ^{2} n\right)$, where $n$ is the number of total simulation samples, and have a second-order accuracy with regard to the sample size (the inverse simulation bandwidth). Because of the lower complexity of these methods, they are often deemed to be the best candidates for implementation in real transmission systems, where the accuracy of these methods will eventually transform into the accuracy of a future instrumental DSP implementation of the (I)NFT.

A quick examination of the Zakharov-Shabat eigenvalue problem [Eq. (3)] brings one to the conclusion that the higher the input power, i.e., the larger the magnitude of $q(t)$, the stiffer the system in Eq. (3) becomes. This means that when one tries to generate a time-domain signal from a multicarrier spectral sequence such as that in Eq. (8), deterioration of the NFT processing occurs due to at least two factors: (i) the large number of subcarriers, leading to a high peak-to-average power ratio (PAPR), and (ii) the large magnitudes of the complex coefficients $c_{k}$. In both cases the observed accuracy of all the existing inverse and direct NFT routines starts to drop [28] (see also the comments on the subject in Ref. [27]). In our simulations, we note that the high PAPR (i) is less of an issue than the overall high power scaling (ii). An additional contributing factor is the size of the processing window (burst width) at the receiver. When processing a noisy input, it is desirable to make the 
processing window as small as possible to make sure that no excess time-domain noise is absorbed and to ensure the highest possible spectral efficiency. In all the simulations in this paper, we pick the value of the burst size to be equal to the maximum pulse spread at $2000 \mathrm{~km}$. However, NFT-generated pulses are known to produce large tails in the time domain [15], and even without inline ASE the truncation of the signal in the time domain due to the finite burst size introduces errors in the nonlinear spectrum (NS). All the above are constituent components of the processing noise $\Gamma_{\text {proc }}$ that exists regardless of the ASE component, and which compound its effects.

In order to study the contribution of the processing noise, we perform a similar set of simulations to those in the previous section but with the inline ASE switched off. The spectrum is still random due to the quasirandom selection of the initial symbols $c_{k}$. Our goal is to compute the corresponding densities and pseudodensities of this process as a function of the same set of parameters $(S, L)$. The results are presented in Fig. 7(a). We quantify the processing noise as follows [cf. Eq. (9)]:

$$
\begin{aligned}
& \mathcal{E}_{\text {proc }, 1}=\frac{1}{W_{\mathrm{NF}}} \int_{-W_{\mathrm{NF}} / 2}^{W_{\mathrm{NF}} / 2} \mathrm{E}\left[\left|\Gamma_{\text {proc }}(\xi)\right|^{2}\right] d \xi, \\
& \mathcal{E}_{\text {proc }, 2}=\frac{1}{W_{\mathrm{NF}}} \int_{-W_{\mathrm{NF}} / 2}^{W_{\mathrm{NF}} / 2} \mathrm{E}\left[\Gamma_{\text {proc }}(\xi)^{2}\right] d \xi,
\end{aligned}
$$

where $\Gamma_{\text {proc }}$ is still recovered from Eq. (5) and the averaging is performed over the initial conditions. Note that unlike the previous simulations, which use normalized densities via Eqs. (6) and (9), the density in Eq. (11) is not normalized, as there is no a priori reason to expect a linear dependence on the distance or a delta-correlation in the nonlinear frequency space.

First, Fig. 7(a) addresses the behavior of the processing noise versus power: we see that the qualitative behavior is virtually the same regardless of the propagation distance, and the error in the density deteriorates quickly at high power in line with the stiffness argument given above (the time and spectral resolution is kept fixed in all the simulations). The pseudocovariance shown in Fig. 7(b) is four orders of magnitude smaller and can be neglected.

Further, it can be seen from Figs. 7(c) and 7(d) that the processing noise does not noticeably depend on the propagation distance in quite a large interval, and not just for the two values considered in Fig. 5(a). The latter can be explained by the fact that processing noise is injected at the transmitter side (INFT error) and once again at the receiver side (NFT error). The former is always fixed for a fixed input power, while the deviations due to the NFT at the receiver cease to depend on the propagation length once the pulse has dispersed enough and the average power (a)

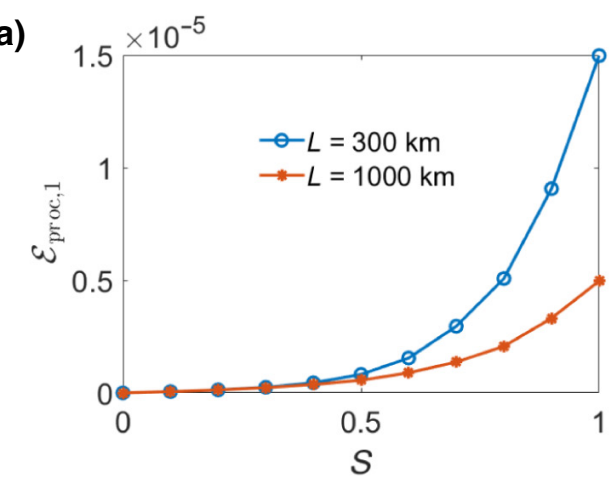

(b)

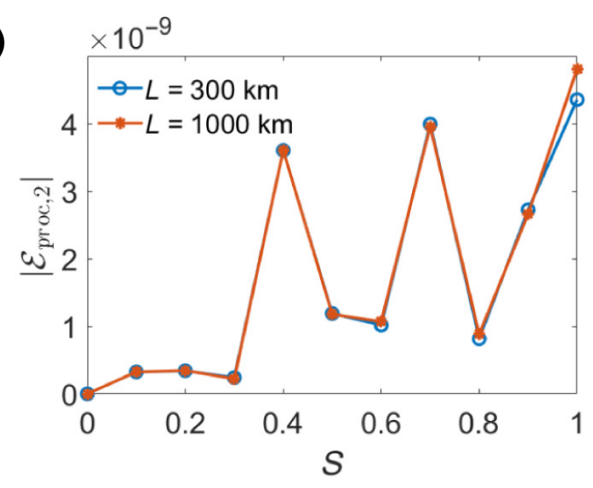

(c)
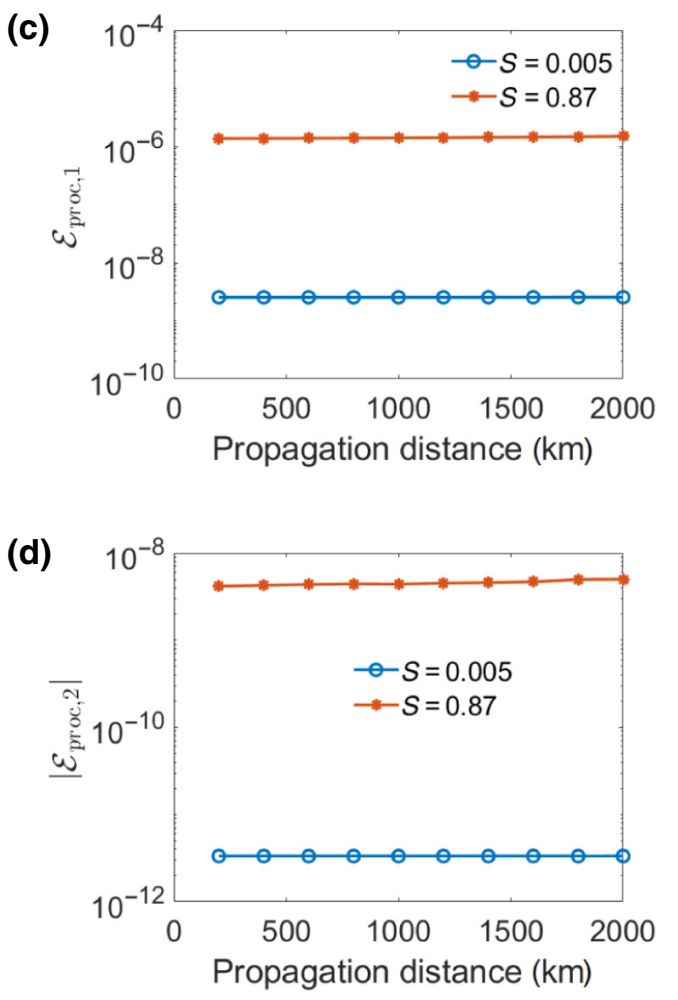

FIG. 7. (a),(b) Dependence of $\left|\mathcal{E}_{i \text {,proc }}\right|$ on the effective power $S$ for 128 subcarriers for the two propagation distances 300 and $1000 \mathrm{~km}$ and for processing noise averaged over initial realization; (c),(d) dependence of processing noise on propagation distance for two different (low and high) signal powers. 
has dropped, which in our system corresponds roughly to $300 \mathrm{~km}$.

\section{Extracting the ASE noise}

Finally, we present a simple method for removing the contribution of the processing noise from the full simulation, the results of which are presented in Fig. 8. Note that we are not referring to some nonlinear filtering procedure [64] that removes the processing noise from the signal - this would require novel stable numerical algorithms or precompensation (see below) - but, rather, we outline a procedure that could help in understanding better the properties of the physical inline ASE noise in the NF domain, $\Gamma$. The idea is based on the simple assumption that the processing and ASE noise have different sources and are independent of each other, and hence their variances should add, at least approximately. Therefore, one can effectively isolate the ASE contribution to the curves
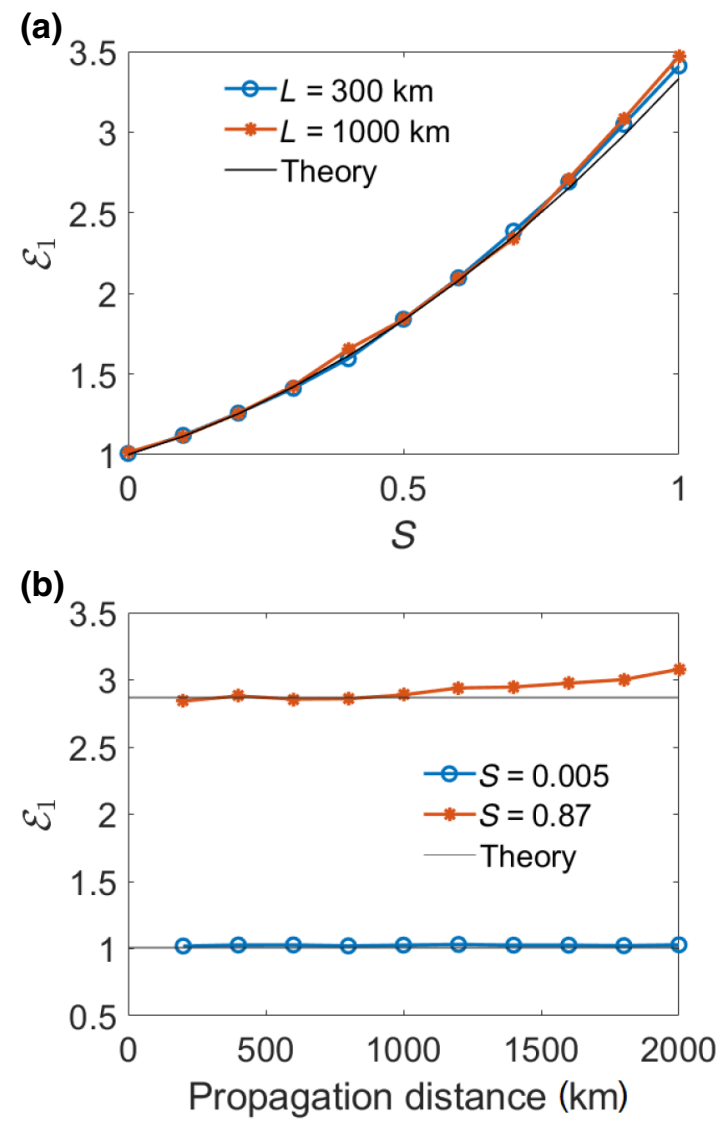

FIG. 8. Dependence of the total power spectral density $\mathcal{E}_{1}$ (after removal of the processing noise) on (a) the effective power $S$ for two propagation distances $L_{1}=300 \mathrm{~km}$ and $L_{2}=1000 \mathrm{~km}$, and (b) on the propagation distance for two different (low and high) values of the effective NF power $S$, for 128 subcarriers. The black line denotes the results obtained with the use of the analytical formula in Eq. (10). for the full simulations presented in Figs. 5 and 6 by subtracting the processing contribution described in the previous subsection [after normalizing it by the distance and the spectral-harmonic spacing $\Delta \xi$ to get the normalized PSD $E_{1}$ from Eq. (6)]. Note that we present only the result for the density $E_{1}$ since, as we see above, the contribution of the processing noise to the pseudodensity is negligible.

It can be seen that after removing the processing noise, our results extracted from the numerical simulations coincide much better with the predictions of the theoretical model for moderate propagation distances, as expected. However, for longer link lengths the theory still underestimates somewhat the level of noise in the system. As mentioned before, this observation is most likely connected to the fact that the theory does not take into account the higher-order contributions that bring about the propagation-distance dependence of the inline covariance, and relies on specific assumptions related to the asymptotic properties of our signals.

In view of the above, we expect that the influence of the processing noise can be mitigated by using half-dispersion precompensation, which makes the pulses both at the transmitter and at the receiver end broader, with lower peak power, thus increasing the accuracy of both NFT operations. However, the details of this question demand a separate study, and we do not address it in the current paper.

\section{CONCLUSIONS}

In this paper, we study the properties of the inline ASE noise and the numerical or processing error translated into the NF domain, for a NFDM transmission system that employs a continuous NF spectrum combined with OFDM-type modulation. We show that the effective nonlinear noise emerging in the NF domain deviates significantly from the progenitor Gaussian ASE noise in the optical domain, displaying a strong dependence on the signal itself. We find that the complex covariance of the NF noise normalized to the propagation distance shows a nonmonotonic dependence on the latter and deviates noticeably from its linear limits. We also underline the deviations of our numerical results from a previous approximate theoretical model that takes into account only the effects of the ASE noise. These deviations are especially pronounced at higher signal power and are explained by the presence of previously unstudied processing noise having an entirely different physical origin. Our results can be used both for the optimization of the performance of NFT-based communication systems and for the improvement of analytical models and approaches referring to the description of NF noise and related information-theory measures. 


\section{ACKNOWLEDGMENTS}

This project received funding from the European Union's Horizon 2020 research and innovation program under the Marie Sklodowska-Curie Grant Agreement, Grants No. 1751561 (MP) and No. 843801 (NBC). S.A.D. was supported by the Israel Science Foundation (Grant No. 466/18). J.E.P. acknowledges support from the Leverhulme Trust, Grant No. RP-2018-063. The authors are grateful to Sander Wahls for his kind assistance with using the fast (I)NFT codes and to Sergei K. Turitsyn for helpful discussions.

\section{APPENDIX A: NORMALIZATION UNITS}

The generalized NLSE in real-world units can be presented as [7]

$$
i \frac{\partial Q}{\partial Z}-\frac{\beta_{2}}{2} \frac{\partial^{2} Q}{\partial T^{2}}+\gamma Q|Q|^{2}=\tilde{\eta}(Z, T),
$$

where $Q(Z, T)$ is a slowly varying complex field envelope, $Z$ stands for the propagation distance along the fiber, and $T$ is the time in a frame comoving with the velocity of the envelope. We are dealing with the anomalous dispersion regime, assuming that the dispersion coefficient is negative, i.e., $\beta_{2}<0$ : a typical value for standard telecom fiber is $\beta_{2} \approx-22 \mathrm{ps}^{2} / \mathrm{km}$. The instantaneous Kerr nonlinearity coefficient $\gamma$ characterizes the nonlinearity strength [a typical value is $\gamma=1.27(\mathrm{~W} \times \mathrm{km})^{-1}$ ]. The model of Eq. (A1) can be applied either to a path-averaging description of a practical fiber-optic system with periodic gain and loss or to a system with ideal Raman amplification (for more details, see Ref. [1,7]). Here, for simplicity, but without loss of generality, we refer to the latter case. The AWGN term $\tilde{\eta}(Z, T)$ is circular delta-correlated Gaussian noise with a power spectral density given by [7]

$$
2 \tilde{D}=h v_{0} K_{T} \chi,
$$

where $\chi$ is the fiber loss coefficient (typically $\chi=0.2$ $\mathrm{dB} / \mathrm{km}$ at the carrier wavelength $\left.\lambda_{0}=1.55 \mu \mathrm{m}\right), K_{T}$ is the temperature-dependent phonon-occupancy factor that characterizes the Raman pump providing the distributed gain (under normal conditions $K_{T}$ is close to unity, i.e., $K_{T}=1.1$ to 1.2 ), and $v_{0}$ is the carrier frequency of the signal corresponding to $\lambda_{0}$, equal to $193.55 \mathrm{THz}$.

In the main text, we use the explicit form of the NFT operations associated with the normalized version of the NLSE, Eq. (1). In our paper, we normalize the time in Eq. (A1) to some characteristic time related to an input signal $T_{0}$. We choose the time normalization $T_{0}$ such that the spacing between the dimensionless nonlinear carriers in Eq. (8) is one-half, which means that in the quasilinear limit the initial pulse width will be exactly $T_{0}$.

Next, the distance is normalized by the dispersion length $Z_{0}=2 T_{0}^{2} /\left|\beta_{2}\right|$. Finally, we measure the power of the field in units of $P_{0}=2 /\left(\gamma Z_{0}\right)$ and normalize the signal amplitude correspondingly. A summary of the normalizations is as follows:

$$
\frac{T}{T_{0}} \rightarrow t, \quad \frac{Z}{Z_{0}} \rightarrow z, \quad \frac{Q}{\sqrt{P_{0}}}=\rightarrow q .
$$

Thus Eq. (A1) is recast in the form of Eq. (1). When proceeding to normalized time, distance, and power, the normalized noise intensity $D$ scales as

$$
D=\tilde{D} \frac{Z_{0}}{P_{0} T_{0}}
$$

The scaling of the dimensionless linear and nonlinear Fourier spectra follows from the above rules.

\section{APPENDIX B: AVERAGING THE PSD OF THE NONLINEAR NOISE OVER THE NONLINEAR BANDWIDTH AND INPUT SYMBOLS}

The nonlinear OFDM spectrum in the NF domain can be written as

$$
r(\xi)=\sum_{k=-N_{\mathrm{ch} / 2}+1}^{N_{\mathrm{ch}} / 2} c_{k} \operatorname{sinc}(\alpha \xi-k),
$$

where $\alpha$ is a frequency-scaling factor and $c_{k}$ are complex independent identically distributed coefficients drawn from a symmetric constellation such as quadrature amplitude modulation or M-ary phase shift keying (M-PSK) so that

$$
\left\langle c_{k} c_{k^{\prime}}\right\rangle=0, \quad\left\langle c_{k} c_{k^{\prime}}^{*}\right\rangle=S \delta_{k k^{\prime}} .
$$

When $\alpha=2$, one recovers Eq. (8).

To obtain the averaged PSD of the nonlinear noise $\mathcal{E}_{1}$ according to Eqs. (7a) and (9), we need to average the quantities $|r(\xi)|^{2}$ and $|r(\xi)|^{4}$. Let us begin with the symbol averaging $\langle\cdots\rangle$. We have

$$
\begin{aligned}
\left\langle|r(\xi)|^{2}\right\rangle= & \sum_{k, k^{\prime}}\left\langle c_{k} c_{k^{\prime}}^{*}\right\rangle \operatorname{sinc}(\alpha \xi-k) \operatorname{sinc}\left(\alpha \xi-k^{\prime}\right) \\
= & S \sum_{k} \operatorname{sinc}^{2}(\alpha \xi-k), \\
\left\langle|r(\xi)|^{4}\right\rangle= & \sum_{k, k^{\prime}} \sum_{m, m^{\prime}}\left\langle c_{k} c_{k^{\prime}}^{*} c_{m} c_{m^{\prime}}^{*}\right\rangle \\
& \times \operatorname{sinc}(\alpha \xi-k) \operatorname{sinc}\left(\alpha \xi-k^{\prime}\right) \\
& \times \operatorname{sinc}(\alpha \xi-m) \operatorname{sinc}\left(\alpha \xi-m^{\prime}\right) \\
= & 2 S^{2} \sum_{k \neq m} \operatorname{sinc}^{2}(\alpha \xi-k) \operatorname{sinc}^{2}(\alpha \xi-m) \\
& +K S^{2} \sum_{k} \operatorname{sinc}^{4}(\alpha \xi-k),
\end{aligned}
$$


where we introduce the kurtosis of the symbol distribution, $K=\left\langle\left|c_{k}\right|^{4}\right\rangle /\left\langle\left|c_{k}\right|^{2}\right\rangle^{2}$. For M-PSK, for example, $K=1$.

Next, we need to average the above over the total nonlinear bandwidth $W_{\mathrm{NF}}=N_{\mathrm{ch}} / \alpha$. Assuming the number of subcarriers to be large and neglecting boundary effects, one can extend the integration over an infinite domain and make use of the following integrals:

$$
\begin{aligned}
\int \operatorname{sinc}^{2} x d x & =1 \\
\int \operatorname{sinc}^{4} x d x & =2 / 3 \\
\int \operatorname{sinc}^{2} x \operatorname{sinc}^{2}(x+\Delta k) d x & =\frac{1}{\pi^{2} \Delta k^{2}}, \quad \Delta k= \pm 1,2,3, \ldots
\end{aligned}
$$

Using the above, we obtain

$$
\begin{aligned}
\mathcal{E}_{1} & =1+S+\frac{2 S^{2}}{\pi^{2} N_{\mathrm{ch}}} \sum_{k \neq m} \frac{1}{(m-k)^{2}}+\frac{2 K S^{2}}{3} \\
& =1+S+\frac{4 S^{2}}{\pi^{2} N_{\mathrm{ch}}} \sum_{k=1}^{N_{\mathrm{ch}}-1} \frac{k}{\left(N_{\mathrm{ch}}-k\right)^{2}}+\frac{2 K S^{2}}{3} .
\end{aligned}
$$

The sum in the third term can be transformed as

$$
\frac{1}{N_{\mathrm{ch}}} \sum_{k=1}^{N_{\mathrm{ch}}-1} \frac{k}{\left(N_{\mathrm{ch}}-k\right)^{2}}=\sum_{k=1}^{N_{\mathrm{ch}}-1} \frac{1}{k^{2}}-\frac{1}{N_{\mathrm{ch}}} \sum_{k=1}^{N_{\mathrm{ch}}-1} \frac{1}{k} .
$$

The last term in the above behaves asymptotically as $\ln N_{\text {ch }} / N_{\text {ch }}$ and, therefore, can be neglected. In the first term, at large $N_{\text {ch }}$ one can extend the sum to infinity and obtain the result equal to $\zeta(2)=\pi^{2} / 6$, where $\zeta(n)$ is the Riemann zeta function. Connecting all the above together, we obtain the final formula valid for a uniform symbol distribution over an arbitrary constellation,

$$
\mathcal{E}_{1}=1+S+\frac{2}{3}(K+1) S^{2},
$$

which in the case of QPSK $(K=1)$ specializes to Eq. (10).

[1] G. P. Agrawal, Fiber-Optic Communication Systems (John Wiley \& Sons, Hoboken, New Jersey, 2012), Vol. 222.

[2] Odyssey of Light in Nonlinear Optical Fibers: Theory and Applications, edited by K. Porsezian and R. Ganapathy, (CRC Press, Boca Raton, 2017).

[3] A. Ellis, M. McCarthy, M. Al Khateeb, M. Sorokina, and N. Doran, Performance limits in optical communications due to fiber nonlinearity, Adv. Opt. Photonics 9, 429 (2017).

[4] E. Agrell, M. Karlsson, A. Chraplyvy, D. J. Richardson, P. M. Krummrich, P. Winzer, K. Roberts, J. K. Fischer, S. J. Savory, and B. J. Eggleton et al. Roadmap of optical communications, J. Opt. 18, 063002 (2016).
[5] P. J. Winzer, D. T. Neilson, and A. R. Chraplyvy, Fiber-optic transmission and networking: The previous 20 and the next 20 years, Opt. Express 26, 24190 (2018).

[6] M. Stephens, V. Gordienko, and N. Doran, $20 \mathrm{db}$ net-gain polarization-insensitive fiber optical parametric amplifier with $>2$ THz bandwidth, Opt. Express 25, 10597 (2017).

[7] R.-J. Essiambre, G. Kramer, P. J. Winzer, G. J. Foschini, and B. Goebel, Capacity limits of optical fiber networks, J. Lightwave Technol. 28, 662 (2010).

[8] E. Ip and J. Kahn, Compensation of dispersion and nonlinear impairments using digital backpropagation, J. Lightwave Technol. 26, 3416 (2008).

[9] E. Giacoumidis, I. Aldaya, M. A. Jarajreh, A. Tsokanos, S. T. Le, F. Farjady, Y. Jaouën, A. D. Ellis, and N. J. Doran, Volterra-based reconfigurable nonlinear equalizer for coherent OFDM, IEEE. Photonics Technol. Lett. 26, 1383 (2014).

[10] L. B. Du, M. M. Morshed, and A. J. Lowery, Fiber nonlinearity compensation for OFDM super-channels using optical phase conjugation, Opt. Express 20, 19921 (2012).

[11] L. B. Y. Du and A. J. Lowery, Phase-conjugated twin waves for communication beyond the kerr nonlinearity limit, Nat. Photonics 7, 560 (2013).

[12] J. C. Cartledge, F. P. Guiomar, F. R. Kschischang, G. Liga, and M. P. Yankov, Digital signal processing for fiber nonlinearities, Opt. Express 25, 1916 (2017).

[13] A. Hasegawa and T. Nyu, Eigenvalue communication, J. Lightwave Technol. 11, 395 (1993).

[14] M. Yousefi and F. Kschischang, Information transmission using the nonlinear Fourier transform, part I-III, IEEE Trans. Inf. Theory 60, 4312 (2014).

[15] J. E. Prilepsky, S. A. Derevyanko, K. J. Blow, I. Gabitov, and S. K. Turitsyn, Nonlinear Inverse Synthesis and Eigenvalue Division Multiplexing in Optical Fiber Channels, Phys. Rev. Lett. 113, 013901 (2014).

[16] S. Le, V. Aref, and H. Buelow, Nonlinear signal multiplexing for communication beyond the Kerr nonlinearity limit, Nat. Photonics 11, 570 (2017).

[17] S. K. Turitsyn, J. E. Prilepsky, S. T. Le, S. Wahls, L. L. Frumin, M. Kamalian, and S. A. Derevyanko, Nonlinear Fourier transform for optical data processing and transmission: Advances and perspectives, Optica 4, 307 (2017).

[18] S. Gaiarin, A. M. Perego, E. P. da Silva, F. Da Ros, and D. Zibar, Dual-polarization nonlinear Fourier transform-based optical communication system, Optica 5, 263 (2018).

[19] F. Da Ros, S. Civelli, S. Gaiarin, E. P. da Silva, N. De Renzis, M. Secondini, and D. Zibar, Dual-polarization NFDM transmission with continuous and discrete spectral modulation, J. Lightwave Technol. 37, 2335 (2019).

[20] X. Yangzhang, V. Aref, S. T. Le, H. Buelow, D. Lavery, and P. Bayvel, Dual-polarization non-linear frequency-division multiplexed transmission with $b$-modulation, J. Lightwave Technol. 37, 1570 (2019).

[21] S. T. Le, 2019 24th OptoElectronics and Communications Conference (OECC) and 2019 International Conference on Photonics in Switching and Computing (PSC) (2019), pp. 1-3.

[22] V. Zakharov and A. Shabat, Exact theory of twodimensional self-focusing and one-dimensional self- 
modulation of waves in nonlinear media, Sov. Phys. JETP 34, 62 (1972).

[23] M. J. Ablowitz and H. Segur, Solitons and the Inverse Scattering Transform (SIAM, Philadelphia, 1981), Vol. 4.

[24] A. Hasegawa and Y. Kodama, Solitons in Optical Communications (Oxford University Press, USA, 1995), Vol. 7.

[25] S. Wahls, S. T. Le, J. E. Prilepsky, H. V. Poor, and S. K. Turitsyn, 2015 IEEE 16th International Workshop on Signal Processing Advances in Wireless Communications (SPAWC) (IEEE, 2015), pp. 445-449.

[26] E. V. Sedov, A. A. Redyuk, M. P. Fedoruk, A. A. Gelash, L. L. Frumin, and S. K. Turitsyn, Soliton content in the standard optical OFDM signal, Opt. Lett. 43, 5985 (2018).

[27] S. Wahls, S. Chimmalgi, and P. J. Prins, Optical Fiber Communication Conference (Optical Society of America, 2019), pp. W2A-50.

[28] I. T. Lima, T. D. DeMenezes, V. S. Grigoryan, M. O'Sullivan, and C. R. Menyuk, Nonlinear compensation in optical communications systems with normal dispersion fibers using the nonlinear Fourier transform, J. Lightwave Technol. 35, 5056 (2017).

[29] S. T. Le, J. E. Prilepsky, and S. K. Turitsyn, Nonlinear inverse synthesis technique for optical links with lumped amplification, Opt. Express 23, 8317 (2015).

[30] M. Kamalian, J. E. Prilepsky, S. T. Le, and S. K. Turitsyn, On the design of NFT-based communication systems with lumped amplification, J. Lightwave Technol. 35, 5464 (2017).

[31] S. T. Le, J. E. Prilepsky, P. Rosa, J. D. Ania-Castañón, and S. K. Turitsyn, Nonlinear inverse synthesis for optical links with distributed raman amplification, J. Lightwave Technol. 34, 1778 (2015).

[32] S. Gaiarin, F. Da Ros, N. De Renzis, E. P. da Silva, and D. Zibar, Dual-polarization NFDM transmission using distributed Raman amplification and NFT-domain equalization, IEEE Photonics Technol. Lett. 30, 1983 (2018).

[33] E. Iannoe, F. Matera, A. Mecozzi, and M. Settembre, Nonlinear Optical Communication Networks (John Wiley \& Sons, New York, 1998).

[34] J. P. Gordon and H. A. Haus, Random walk of coherently amplified solitons in optical fiber transmission, Opt. Lett. 11, 665 (1986).

[35] S. A. Derevyanko, S. Turitsyn, and D. Yakushev, Nongaussian statistics of an optical soliton in the presence of amplified spontaneous emission, Opt. Lett. 28, 2097 (2003).

[36] S. A. Derevyanko, S. K. Turitsyn, and D. A. Yakushev, Fokker-planck equation approach to the description of soliton statistics in optical fiber transmission systems, JOSA B 22, 743 (2005).

[37] Q. Zhang and F. R. Kschischang, Improved soliton amplitude estimation via the continuous spectrum, J. Lightwave Technol. 37, 3087 (2019).

[38] Q. Zhang and T. H. Chan, 2015 IEEE 16th International Workshop on Signal Processing Advances in Wireless Communications (SPAWC) (IEEE, 2015), pp. 455-459.

[39] W. Q. Zhang, T. Gui, Q. Zhang, C. Lu, T. M. Monro, T. H. Chan, A. P. T. Lau, and V. S. Afshar, Correlated eigenvalues of multi-soliton optical communications, Sci. Rep. 9, 6399 (2019).

[40] J. E. Prilepsky, S. A. Derevyanko, and S. K. Turitsyn, Optical Communication Systems: Limits and Possibilities, edited by A. Ellis and M. Sorokina (Jenny Stanford Publishing, 2019), Chap. 7, pp. 243-272.

[41] S. Wahls, SCC 2017; 11th International ITG Conference on Systems, Communications and Coding (VDE, 2017), pp. $1-6$.

[42] S. A. Derevyanko and J. E. Prilepsky, Random input problem for the nonlinear Schrödinger equation, Phys. Rev. E 78, 046610 (2008).

[43] S. A. Derevyanko, J. E. Prilepsky, and S. K. Turitsyn, Capacity estimates for optical transmission based on the nonlinear Fourier transform, Nature Commun. 7, 307 (2016).

[44] I. Tavakkolnia and M. Safari, Capacity analysis of signaling on the continuous spectrum of nonlinear optical fibers, J. Lightwave Technol. 35, 2086 (2017).

[45] S. Civelli, E. Forestieri, and M. Secondini, Why noise and dispersion may seriously hamper nonlinear frequencydivision multiplexing, IEEE Photonics Technol. Lett. 29, 1332 (2017).

[46] S. T. Le, K. Schuh, F. Buchali, and H. Buelow, Optical Fiber Communication Conference (Optical Society of America, 2018), pp. W1G-6.

[47] S. T. Le and H. Buelow, Photonic Networks; 19th ITGSymposium (VDE, 2018), pp. 1-6.

[48] M. Pankratova, A. Vasylchenkova, J. E. Prilepsky, and S. A. Derevyanko, Frontiers in Optics (Optical Society of America, 2018), pp. JW3A-83.

[49] M. L. Pankratova, A. Vasylchenkova, J. Prilepsky, and S. A. Derevyanko, 2018 IEEE British and Irish Conference on Optics and Photonics (BICOP) (2018), pp. 1-3.

[50] M. Pankratova, A. Vasylchenkova, and J. E. Prilepsky, The International Conference on Optical Communication Systems (2019), pp. 1-6.

[51] J. W. Goossens, M. I. Yousefi, Y. Jaouan, and H. Hafermann, Polarization-division multiplexing based on the nonlinear Fourier transform, Opt. Express 25, 26437 (2017).

[52] S. Civelli, S. Turitsyn, M. Secondini, and J. Prilepsky, Polarization-multiplexed nonlinear inverse synthesis with standard and reduced-complexity NFT processing, Opt. Express 26, 17360 (2018).

[53] S. T. Le, J. E. Prilepsky, and S. K. Turitsyn, Nonlinear inverse synthesis for high spectral efficiency transmission in optical fibers, Opt. Express 22, 26270 (2014).

[54] S. Wahls, 2017 European Conference on Optical Communication (ECOC) (2017), pp. 1-3.

[55] T. Gui, G. Zhou, C. Lu, A. P. T. Lau, and S. Wahls, Nonlinear frequency division multiplexing with b-modulation: Shifting the energy barrier, Opt. Express 26, 27978 (2018).

[56] I. Tavakkolnia and M. Safari, 2016 Conference on Lasers and Electro-Optics (CLEO) (IEEE, 2016), pp. 1-2.

[57] V. Aref, S. Le, and H. Buelow, Modulation over nonlinear Fourier spectrum: Continuous and discrete spectrum, J. Lightwave Technol. 36, 1289 (2018).

[58] S. Wahls and H. V. Poor, Fast numerical nonlinear Fourier transforms, IEEE Trans. Inform. Theory 61, 6957 (2015). 
[59] V. E. Zakharov and S. V. Manakov, Asymptotic behavior of non-linear wave systems integrated by the inverse scattering method, Sov. Phys. JETP 44, 106 (1976).

[60] S. Wahls and H. V. Poor, 2013 IEEE International Conference on Acoustics, Speech and Signal Processing (IEEE, 2013), pp. 5780-5784.

[61] S. Wahls and V. Vaibhav, Fast inverse nonlinear Fourier transforms for continuous spectra of Zakharov-Shabat type, arXiv:1607.01305.
[62] V. Vaibhav and S. Wahls, 2017 Optical Fiber Communications Conference and Exhibition (OFC) (IEEE, 2017), pp. $1-3$.

[63] S. Wahls, S. Chimmalgi, and P. J. Prins, Fnft: A software library for computing nonlinear Fourier transforms, J. Open Source Softw. 3, 597 (2018).

[64] M. Kamalian, J. E. Prilepsky, S. A. Derevyanko, S. T. Le, and S. K. Turitsyn, Conference on Lasers and ElectroOptics (Optical Society of America, 2017), p. JTh2A.135. 